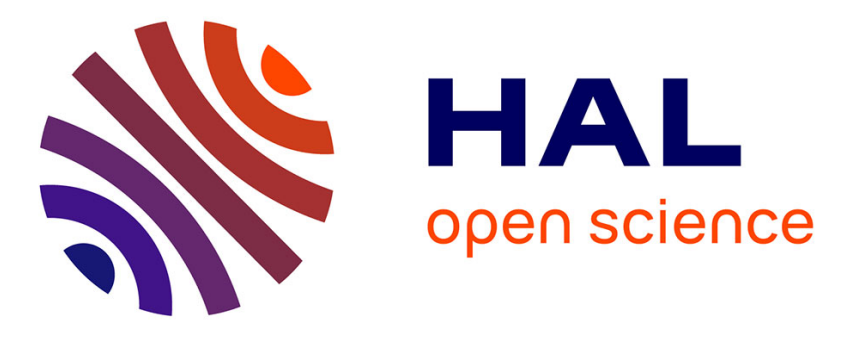

\title{
Robust stability under relaxed persistent excitation conditions
}

\author{
Denis Efimov, Nikita Barabanov, Roméo Ortega
}

\section{To cite this version:}

Denis Efimov, Nikita Barabanov, Roméo Ortega. Robust stability under relaxed persistent excitation conditions. 57th IEEE Conference on Decision and Control (CDC 2018), Dec 2018, Miami Beach, FL, United States. hal-01888575

\section{HAL Id: hal-01888575 \\ https://hal.inria.fr/hal-01888575}

Submitted on 5 Oct 2018

HAL is a multi-disciplinary open access archive for the deposit and dissemination of scientific research documents, whether they are published or not. The documents may come from teaching and research institutions in France or abroad, or from public or private research centers.
L'archive ouverte pluridisciplinaire HAL, est destinée au dépôt et à la diffusion de documents scientifiques de niveau recherche, publiés ou non, émanant des établissements d'enseignement et de recherche français ou étrangers, des laboratoires publics ou privés. 


\title{
Robust stability under relaxed persistent excitation conditions
}

\author{
D. Efimov, N. Barabanov, R. Ortega
}

\begin{abstract}
For linear time-varying systems with a persistently excited state matrix it is well-known that input-to-state stability is recovered. In this note a relaxed condition on persistence of excitation is studied together with related robust stability notions (input-to-state stability and integral input-to-state stability). The results are illustrated by simulations for scalar systems.
\end{abstract}

\section{INTRODUCTION}

The problems of stability and robust stability are one of the main issues studied in the theory of control. There are some special systems related to benchmark models or important problem statements whose stability has been analyzed in detail. One of such a problem deals with a time-varying linear dynamical system of the following form:

$$
\begin{gathered}
\dot{p}(t)=-\gamma R(t) R^{\top}(t) p(t)+b(t), t \geq t_{0}, \\
p\left(t_{0}\right) \in \mathbb{R}^{l}, t_{0} \in \mathbb{R},
\end{gathered}
$$

where $p(t) \in \mathbb{R}^{l}$ is the state, the functions $R: \mathbb{R} \rightarrow \mathbb{R}^{l \times k}$ and $b: \mathbb{R} \rightarrow \mathbb{R}^{l}$ are (Lebesgue) measurable and essentially bounded; $\gamma>0$. This problem is related with adaptive estimation and identification of linear regression models [1]. The peculiarity of this system consists in the requirement imposed on the matrix function $R$, which is assumed usually to be persistently excited. It is a well-known fact [2], that if $R$ is persistently excited, then (1) is globally exponentially stable for $b(t) \equiv 0, t \geq t_{0}$ and input-to-state stable (ISS) for any essentially bounded $b$.

Recently, several relaxed excitation notions have been proposed for $R$ in [3], [4] implying global (not uniform) asymptotic stability of (1). The goal of this note is to compare them and to establish relations with ISS and integral ISS (iISS) properties introduced in [5], [6].

The paper outline is as follows. Preliminaries (persistence of excitation and stability notions) are introduced in Section

D. Efimov is with Inria, Univ. Lille, CNRS, UMR 9189 - CRIStAL, F59000 Lille, France.

N. Barabanov is with Department of Mathematics, North Dakota State University, Fargo, ND 58102, USA.

R. Ortega is with Laboratoire des Signaux et Systémes, École Supérieure d'Electricité (SUPELEC), Gif-sur-Yvette 91192, France.

D. Efimov and N. Barabanov are with Department of Control Systems and Informatics, University ITMO, 49 av. Kronverkskiy, 197101 Saint Petersburg, Russia.

The results of Section V were supported solely by the Russian Federation President Grant (No. 14.W01.16.6325-MD (MD-6325.2016.8)). The other research was partially supported by the Government of Russian Federation (Grant 08-08) and the Ministry of Education and Science of Russian Federation (Project 14.Z50.31.0031).
II. The relaxed excitation notions from [3], [4] are discussed in Section III. Relations with ISS and iISS properties with respect to different perturbations (exogenous disturbances or measurement noises) are considered in Section IV. The scalar case and some results of simulation are presented in Section V. Some concluding remarks are given in Section VI.

\section{Preliminaries}

Denote $\mathbb{R}_{+}=\{x \in \mathbb{R}: x \geq 0\}$, where $\mathbb{R}$ is the set of real numbers, $\mathbb{N}$ is the set of natural numbers. Let $|\cdot|$ denotes the Euclidean norm on $\mathbb{R}^{n}$ (for $n \in \mathbb{N}$ ) or the corresponding induced matrix norm. For a (Lebesgue) measurable and essentially bounded function $b: \mathbb{R} \rightarrow \mathbb{R}^{l}$ denote $\|b\|_{\infty}=\sup _{t \in \mathbb{R}}|b(t)|$.

Denote by $\mathcal{K}^{+}$the class of continuous functions $\alpha: \mathbb{R}_{+} \rightarrow$ $\mathbb{R}_{+}, \alpha \in \mathcal{K}^{+}$belongs to the class $\mathcal{K}$ if $\alpha(0)=0$ and the function is strictly increasing. The function $\alpha \in \mathcal{K}^{+}$ belongs to the class $\mathcal{K}_{\infty}$ if $\alpha \in \mathcal{K}$ and it increases to infinity. A continuous function $\mu: \mathbb{R}_{+} \times \mathbb{R}_{+} \rightarrow \mathbb{R}_{+}$belongs to the class $\mathcal{K} \mathcal{L}$ if $\mu(\cdot, t) \in \mathcal{K}_{\infty}$ for each fixed $t \in \mathbb{R}_{+}$and $\lim _{t \rightarrow+\infty} \mu(s, t)=0$ for each fixed $s \in \mathbb{R}_{+}$.

\section{A. Persistence of excitation condition}

A (Lebesgue) measurable and square integrable matrix function $R: \mathbb{R} \rightarrow \mathbb{R}^{l \times k}$ with the dimension $l \times k$ verifies the $(\ell, \vartheta)$-Persistence of Excitation (PE) condition, if there exist constants $\ell>0$ and $\vartheta>0$ such that

$$
\int_{t}^{t+\ell} R(s) R^{\top}(s) d s \geq \vartheta I_{l}
$$

for any $t \in \mathbb{R}$, where $I_{l}$ denotes the identity matrix of dimension $l \times l$.

Lemma 1. [7] Assume that in the system (1) the function $R$ is continuous, bounded by $\rho=\sup _{t \in \mathbb{R}}|R(t)|<+\infty$, and $(\ell, \vartheta)-P E$ for some $\ell>0, \vartheta>0$. Then, for any $t_{0} \in \mathbb{R}$ and any initial condition $p\left(t_{0}\right) \in \mathbb{R}^{l}$ :

$|p(t)| \leq \sqrt{\zeta} \rho\left[e^{-0.5 \gamma \zeta^{-1}\left(t-t_{0}\right)}\left|p\left(t_{0}\right)\right|+\gamma^{-1} \zeta\|b\|_{\infty}\right] \quad \forall t \geq t_{0}$ for $\zeta=\gamma \eta^{-1} e^{2 \eta \ell}$ and $\eta=-0.5 \ell^{-1} \ln \left(1-\frac{\gamma \vartheta}{1+\gamma^{2} \ell^{2} \rho^{4}}\right)$.

Note that from the definition of PE property, in the conditions of the above lemma,

$$
\vartheta \leq \ell \rho^{2}
$$

then $\frac{\gamma \vartheta}{1+\gamma^{2} \ell^{2} \rho^{4}} \in(0,0.5]$ for any $\gamma>0, \ell>0$ and $\vartheta>0$. Therefore, $\eta$ and $\zeta$ belong to $(0,+\infty)$. 


\section{B. Robust stability properties}

The system (1) is called nonuniform ISS [8] if there exist $\mu \in \mathcal{K} \mathcal{L}, \sigma \in \mathcal{K}^{+}$and $\nu \in \mathcal{K}$ such that for all $t_{0} \in \mathbb{R}$, all $p\left(t_{0}\right) \in \mathbb{R}^{l}$ and (Lebesgue) measurable and essentially bounded $b: \mathbb{R} \rightarrow \mathbb{R}^{l}:$

$$
|p(t)| \leq \mu\left(\sigma\left(t_{0}\right)\left|p\left(t_{0}\right)\right|, t-t_{0}\right)+\nu\left(\|b\|_{\infty}\right) \quad \forall t \geq t_{0} .
$$

The system (1) is called nonuniform iISS [8] if there exist $\mu \in \mathcal{K} \mathcal{L}, \sigma \in \mathcal{K}^{+}, \alpha \in \mathcal{K}_{\infty}$ and $\nu \in \mathcal{K}$ such that for all $t_{0} \in \mathbb{R}$, all $p\left(t_{0}\right) \in \mathbb{R}^{l}$ and (Lebesgue) measurable and integrable $b: \mathbb{R} \rightarrow \mathbb{R}^{l}$ :

$\alpha(|p(t)|) \leq \mu\left(\sigma\left(t_{0}\right)\left|p\left(t_{0}\right)\right|, t-t_{0}\right)+\int_{t_{0}}^{t} \nu(|b(s)|) d s \quad \forall t \geq t_{0}$.

The system (1) is called uniform ISS or iISS if $\sigma(s)=$ const for all $s \in \mathbb{R}$.

\section{RELAXED CONVERGENCE CONDITIONS FOR THE UNFORCED CASE}

First, lest us consider the conditions of asymptotic stability of (1) for the case $b(t) \equiv 0$ for all $t \in \mathbb{R}$. Then, to relax the PE condition we will assume that the parameters $\ell$ and $\vartheta$ are time-dependent:

Assumption 1. Assume that for any $t_{0} \in \mathbb{R}$ there exist sequences of positive numbers $\left\{t_{k}\right\}_{k=0}^{+\infty},\left\{\ell_{k}\right\}_{k=0}^{+\infty}$ and $\left\{\vartheta_{k}\right\}_{k=0}^{+\infty}$ such that for all $k \geq 0$ :

$$
\begin{gathered}
t_{k+1} \geq t_{k}+\ell_{k}, \\
\int_{t_{k}}^{t_{k}+\ell_{k}} R(s) R^{\top}(s) d s \geq \vartheta_{k} I_{l} .
\end{gathered}
$$

There are several results relating this assumption and asymptotic stability of (1):

Proposition 1. [4] Let Assumption 1 be satisfied and

$$
\sum_{k=0}^{+\infty} \frac{\vartheta_{k}}{\gamma^{-1}+\gamma\left(\int_{t_{k}}^{t_{k}+\ell_{k}}|R(t)|^{2} d t\right)^{2}}=+\infty .
$$

Then the system (1) with $b(t) \equiv 0$ for all $t \in \mathbb{R}$ is globally asymptotically stable at the origin.

In [3] another condition of global asymptotic stability (GAS) for (1) has been established, which is based on the result given below that contains a small extension together with a proof:

Proposition 2. Let $b(t) \equiv 0$ for all $t \in \mathbb{R}$ and $\Phi\left(t, t_{0}\right)$ be the transition matrix of (1) satisfying

$\frac{\partial}{\partial t} \Phi\left(t, t_{0}\right)=-\gamma R(t) R^{\top}(t) \Phi\left(t, t_{0}\right), t \geq t_{0}, \Phi\left(t_{0}, t_{0}\right)=I_{l}$,

then for all $t \geq t_{0}$ :

$$
\begin{aligned}
e^{-2 \gamma \int_{t_{0}}^{t}|R(s)|^{2} d s} I_{l} & \leq \Phi^{\top}\left(t, t_{0}\right) \Phi\left(t, t_{0}\right) \\
& \leq I_{l}-\frac{\int_{t_{0}}^{t} R(s) R^{\top}(s) d s}{\gamma^{-1}+\gamma\left(\int_{t_{0}}^{t}|R(s)|^{2} d s\right)^{2}} .
\end{aligned}
$$

Proof. For any $t_{0} \in \mathbb{R}$ and $p\left(t_{0}\right) \in \mathbb{R}^{l}$ denote $p(t)=$ $\Phi\left(t, t_{0}\right) p\left(t_{0}\right)$ (the solution of (1) with initial condition $p\left(t_{0}\right)$ at initial time $t_{0}$ ) and consider an auxiliary Lyapunov function $W(p)=p^{\top} p$, whose derivative for the system (1) has the form:

$$
\begin{aligned}
\frac{d}{d t} W(p(t)) & =-2 \gamma p^{\top}(t) R(t) R^{\top}(t) p(t) \\
& =-2 \gamma\left|R^{\top}(t) p(t)\right|^{2}
\end{aligned}
$$

then

implying

$$
\frac{d}{d t} W(p(t)) \geq-2 \gamma|R(t)|^{2} W(p(t))
$$

$$
W(p(t)) \geq e^{-2 \gamma \int_{t_{0}}^{t}|R(r)|^{2} d r} W\left(p\left(t_{0}\right)\right),
$$

which can be interpreted as

$p^{\top}\left(t_{0}\right) \Phi^{\top}\left(t, t_{0}\right) \Phi\left(t, t_{0}\right) p\left(t_{0}\right) \geq p^{\top}\left(t_{0}\right) e^{-2 \gamma \int_{t_{0}}^{t}|R(r)|^{2} d r} p\left(t_{0}\right)$ providing the required lower estimate on $\Phi^{\top}\left(t, t_{0}\right) \Phi\left(t, t_{0}\right)$ due to an arbitrary choice of $p\left(t_{0}\right)$. From another side, since $0.5|a|^{2} \leq|b|^{2}+|a-b|^{2}$ for any $a, b \in \mathbb{R}^{l}$, for $a=R^{\top}(t) p\left(t_{0}\right)$ and $b=R^{\top}(t) p(t)$ we obtain:

$$
0.5\left|R^{\top}(t) p\left(t_{0}\right)\right|^{2} \leq\left|R^{\top}(t) p(t)\right|^{2}+\left|R^{\top}(t)\left(p\left(t_{0}\right)-p(t)\right)\right|^{2} .
$$

Note that

$$
\begin{gathered}
\left|R^{\top}(t)\left(p(t)-p\left(t_{0}\right)\right)\right|^{2}=\left|R^{\top}(t) \int_{t_{0}}^{t} \dot{p}(s) d s\right|^{2} \\
=\gamma^{2} \int_{t_{0}}^{t} p^{\top}(s) R(s) R^{\top}(s) d s R(t) R^{\top}(t) \int_{t_{0}}^{t} R(s) R^{\top}(s) p(s) d s \\
\leq \gamma^{2}|R(t)|^{2} \int_{t_{0}}^{t} p^{\top}(s) R(s) R^{\top}(s) d s \int_{t_{0}}^{t} R(s) R^{\top}(s) p(s) d s \\
\leq \gamma^{2}|R(t)|^{2}\left(\int_{t_{0}}^{t}|R(s)|\left|R^{\top}(s) p(s)\right| d s\right)^{2} \\
\leq \gamma^{2}|R(t)|^{2} \int_{t_{0}}^{t}|R(s)|^{2} d s \int_{t_{0}}^{t}\left|R^{\top}(s) p(s)\right|^{2} d s
\end{gathered}
$$

where the Cauchy-Schwarz inequality has been used on the last step. Integrating the obtained inequality we conclude that

$$
\begin{aligned}
& \int_{t_{0}}^{t} \mid R^{\top}(s)(p(s)\left.-p\left(t_{0}\right)\right)\left.\right|^{2} d s \leq \int_{t_{0}}^{t} \gamma^{2}|R(r)|^{2} \int_{t_{0}}^{r}|R(s)|^{2} d s \\
& \times \int_{t_{0}}^{r}\left|R^{\top}(s) p(s)\right|^{2} d s d r \\
& \leq \gamma^{2}\left(\int_{t_{0}}^{t}|R(s)|^{2} d s\right)^{2} \int_{t_{0}}^{t}\left|R^{\top}(s) p(s)\right|^{2} d s
\end{aligned}
$$

Next, integrating (4) the following relation is derived:

$$
\begin{gathered}
0.5 \int_{t_{0}}^{t}\left|R^{\top}(s) p\left(t_{0}\right)\right|^{2} d s \leq \int_{t_{0}}^{t}\left|R^{\top}(s) p(s)\right|^{2} d s \\
+\int_{t_{0}}^{t}\left|R^{\top}(s)\left(p(s)-p\left(t_{0}\right)\right)\right|^{2} d s \\
\leq\left[1+\gamma^{2}\left(\int_{t_{0}}^{t}|R(s)|^{2} d s\right)^{2}\right] \int_{t_{0}}^{t}\left|R^{\top}(s) p(s)\right|^{2} d s .
\end{gathered}
$$


Finally, integrating (3) we obtain:

$$
\begin{gathered}
W(p(t))-W\left(p\left(t_{0}\right)\right)=-2 \gamma \int_{t_{0}}^{t}\left|R^{\top}(s) p(s)\right|^{2} d s \\
\leq-\frac{\gamma \int_{t_{0}}^{t}\left|R^{\top}(s) p\left(t_{0}\right)\right|^{2} d s}{1+\gamma^{2}\left(\int_{t_{0}}^{t}|R(s)|^{2} d s\right)^{2}},
\end{gathered}
$$

or equivalently,

$$
\begin{gathered}
p^{\top}\left(t_{0}\right) \Phi^{\top}\left(t, t_{0}\right) \Phi\left(t, t_{0}\right) p\left(t_{0}\right) \\
\leq p^{\top}\left(t_{0}\right)\left(I_{l}-\frac{\gamma \int_{t_{0}}^{t} R(s) R^{\top}(s) d s}{1+\gamma^{2}\left(\int_{t_{0}}^{t}|R(s)|^{2} d s\right)^{2}}\right) p\left(t_{0}\right),
\end{gathered}
$$

and since this inequality is satisfied for any $p\left(t_{0}\right) \in \mathbb{R}^{l}$, the desired upper estimated is substantiated.

This proposition provides generic properties of the transition matrix of (1), from which the stability conditions can be deduced:

Corollary 1. Let Assumption 1 be satisfied and

$$
\prod_{k=0}^{+\infty}\left(1-\frac{\vartheta_{k}}{\gamma^{-1}+\gamma\left(\int_{t_{k}}^{t_{k}+\ell_{k}}|R(t)|^{2} d t\right)^{2}}\right)=0
$$

Then the system (1) with $b(t) \equiv 0$ for all $t \in \mathbb{R}$ is globally asymptotically stable at the origin.

Further, almost all the proofs are omitted for brevity of the presentation.

Remark 1. Since $0<\vartheta_{k} \leq \int_{t_{k}}^{t_{k}+\ell_{k}}|R(t)|^{2} d t$, $\sup _{x \geq 0} \frac{x}{1+x^{2}}=0.5$ and $\ln (1-s) \leq-s$ for $0<s<1$, by applying $\ln (\cdot)$ to both sides of (5) we conclude:

$$
\begin{aligned}
-\infty & =\sum_{k=0}^{+\infty} \ln \left(1-\frac{\vartheta_{k}}{\gamma^{-1}+\gamma\left(\int_{t_{k}}^{t_{k}+\ell_{k}}|R(t)|^{2} d t\right)^{2}}\right) \\
& \leq-\sum_{k=0}^{+\infty} \frac{\vartheta_{k}}{\gamma^{-1}+\gamma\left(\int_{t_{k}}^{t_{k}+\ell_{k}}|R(t)|^{2} d t\right)^{2}}
\end{aligned}
$$

and

$$
\sum_{k=0}^{+\infty} \frac{\vartheta_{k}}{\gamma^{-1}+\gamma\left(\int_{t_{k}}^{t_{k}+\ell_{k}}|R(t)|^{2} d t\right)^{2}} \leq+\infty
$$

which implies (2). And vice versa, the estimate (2) also implies (5), since using the inequality $1-x \leq e^{-x}$ that is satisfied for all $x \in \mathbb{R}$, and by applying $e^{-(\cdot)}$ to both sides of (2) we obtain:

$$
\begin{gathered}
0=e^{-\sum_{k=0}^{+\infty} \frac{\vartheta_{k}}{\gamma^{-1}+\gamma\left(\int_{t_{k}}^{t_{k}+\ell_{k}}|R(t)|^{2} d t\right)^{2}}} \\
\left.=\prod_{k=0}^{+\infty} e^{-\frac{\vartheta_{k}}{\gamma^{-1}+\gamma\left(\int_{t_{k}}^{t_{k}+\ell_{k}}|R(t)|^{2} d t\right)^{2}}}\right) \geq 0 . \\
\geq \prod_{k=0}^{+\infty}\left(1-\frac{\vartheta_{k}}{\gamma^{-1}+\gamma\left(\int_{t_{k}}^{t_{k}+\ell_{k}}|R(t)|^{2} d t\right)^{2}}\right)
\end{gathered}
$$

Thus, the conditions (2) and (5) are equivalent.

\section{ROBUSTNESS WITH RESPECT TO EXTERNAL INPUTS}

Now, let us return to stability analysis of the perturbed system (1) with $b(t) \neq 0$.

Proposition 3. Consider the system (1), then for any $t_{0} \in \mathbb{R}$ and any $p\left(t_{0}\right) \in \mathbb{R}^{l}$

$$
\begin{aligned}
|p(t)| \leq & \sqrt{\left|I_{l}-\frac{\int_{t_{0}}^{t} R(s) R^{\top}(s) d s}{\gamma^{-1}+\gamma\left(\int_{t_{0}}^{t}|R(s)|^{2} d s\right)^{2}}\right|} \\
& \times\left(\left|p\left(t_{0}\right)\right|+e^{\gamma \int_{t_{0}}^{t}|R(s)|^{2} d s} \int_{t_{0}}^{t}|b(s)| d s\right)
\end{aligned}
$$

for all $t \geq t_{0}$.

The latter result provides a generic auxiliary estimate on solutions of (1), which is used next to derive the stability conditions. To this end introduce a sequence

$$
\rho_{k}=\int_{t_{k}}^{t_{k+1}}|R(t)|^{2} d t \quad \forall k \geq 0 .
$$

Theorem 1. Consider the system (1) satisfying Assumption 1 with an additional constraint:

$$
t_{k+1}=t_{k}+\ell_{k}
$$

for all $k \geq 0$, and assume that the property (5) is valid.

$$
\text { If }
$$

$$
\begin{gathered}
\sup _{k \geq 0}\left\{\ell_{k} e^{\gamma \rho_{k}}+\sum_{i=0}^{k} \prod_{j=i}^{k} \sqrt{1-\frac{\vartheta_{j}}{\gamma^{-1}+\gamma \rho_{j}^{2}}} \ell_{i} e^{\gamma \rho_{i}}\right\} \\
\leq \eta_{t_{0}}<+\infty
\end{gathered}
$$

then for each $t_{0} \in \mathbb{R}$ there exists a function $\varrho_{t_{0}} \in \mathcal{K} \mathcal{L}$ such that for all $p\left(t_{0}\right) \in \mathbb{R}^{l}$ :

$$
|p(t)| \leq \varrho_{t_{0}}\left(\left|p\left(t_{0}\right)\right|, t-t_{0}\right)+\eta_{t_{0}}\|b\|_{\infty} \quad \forall t \geq t_{0} .
$$

If

$$
\sup _{k \geq 0} e^{\gamma \rho_{k}} \leq \beta_{t_{0}}<+\infty,
$$

then for each $t_{0} \in \mathbb{R}$ there exists a function $\varrho_{t_{0}} \in \mathcal{K} \mathcal{L}$ such that for all $p\left(t_{0}\right) \in \mathbb{R}^{l}$ :

$$
|p(t)| \leq \varrho_{t_{0}}\left(\left|p\left(t_{0}\right)\right|, t-t_{0}\right)+\beta_{t_{0}} \int_{t_{0}}^{t}|b(t)| d t \quad \forall t \geq t_{0} .
$$


Thus, the conditions of non-uniform ISS property (6) are rather sophisticated and discussed below, while it is worth to stress here that the swap "conditions" of non-uniform iISS property (7) are very weak and satisfied, for example, if the system (1) is asymptotically stable for $b=0$ and

$$
\int_{-\infty}^{+\infty}|R(t)|^{2} d t<+\infty
$$

or

$$
\sup _{k \geq 0} \ell_{k}<+\infty, \text { ess } \sup _{t \geq 0}|R(t)|<+\infty \text {. }
$$

\section{A. Relation with PE conditions}

Note that dependence of $\eta_{t_{0}}$ on the initial time $t_{0} \in \mathbb{R}$ is important in the formulation of Theorem 1 , since if a uniform result would be obtained, i.e. there exist $\bar{\eta} \in \mathbb{R}_{+}$such that $\sup _{t_{0} \in \mathbb{R}} \eta_{t_{0}} \leq \bar{\eta}$, then it implies global exponentially stability (GES) of (1) (and, consequently, fulfillment of PE condition [9]), as it is shown in the following lemma:

Lemma 2. Assume that for the system (1) there exist constants $C_{1} \geq 1$ and $C_{2}>0$ such that for all $t_{0} \in \mathbb{R}$ and all initial conditions $p\left(t_{0}\right) \in \mathbb{R}^{l}$ we have

$$
|p(t)| \leq C_{1}\left|p\left(t_{0}\right)\right|+C_{2}\|b\|_{\infty} \quad \forall t \geq t_{0} .
$$

Then the system (1) with $b(t) \equiv 0$ for all $t \in \mathbb{R}$ is GES.

Proof. Pick up a number $\epsilon \in\left(0,1 / C_{2}\right)$ and set $C_{3}=\frac{2 C_{1}}{1-\epsilon C_{2}}$. Consider a solution $p(t)$ of the system (1) with $\left|p\left(t_{0}\right)\right|=1$ and $b(t) \equiv 0$ for all $t \geq t_{0}$. It is sufficient to prove that function $y(t)=e^{\epsilon\left(t-t_{0}\right)} p(t)$ is bounded. Function $y(t)$ satisfies equation

$$
\dot{y}(t)=-\gamma R(t) R(t)^{T} y(t)+\epsilon y(t) .
$$

We will prove that $|y(t)|<C_{3}$ for all $t \geq t_{0}$. Recall that $C_{1} \geq 1$ and, therefore, $C_{3}>1$. Since $\left|y\left(t_{0}\right)\right|=1$, we have $|y(t)|<C_{3}$ for all sufficiently small $t \geq t_{0}$. Assume, on the contrary to the claim existence of $T>t_{0}$ such that $|y(T)|=$ $C_{3}$. Choose the first $T$ satisfying this property. Denote $b(t)=$ $\epsilon y(t)$ on $\left[t_{0}, T\right]$. Then according to the assumption

$$
|y(t)| \leq C_{1}+C_{2} \epsilon \sup _{t \in\left[t_{0}, T\right]}|y(t)|
$$

for all $t \in\left[t_{0}, T\right]$. Therefore,

$|y(t)| \leq C_{1}+C_{2} \epsilon C_{3}=C_{1}\left(1+\frac{2 \epsilon C_{2}}{1-\epsilon C_{2}}\right)=C_{1} \frac{1+\epsilon C_{2}}{1-\epsilon C_{2}}<C_{3}$

for all $t \in\left[t_{0}, T\right]$. The contradiction at $t=T$ shows that $|y(t)|<C_{3}$ for all $t \geq t_{0}$, which completes the proof.

Corollary 2. If system (1) with $b(t) \equiv 0$ for all $t \in \mathbb{R}$ is not GES, then the assumption of Lemma 2 is not satisfied, and therefore the system (1) is not ISS.

The proof of Theorem 1 shows that for every $t_{0} \in \mathbb{R}$ function $\rho_{t_{0}}\left(s, t-t_{0}\right)$ in (6) may be chosen as $\rho_{t_{0}}\left(s, t-t_{0}\right)=$ $s k_{t_{0}}\left(t-t_{0}\right)$ with a decreasing function $k_{t_{0}}: \mathbb{R}_{+} \rightarrow \mathbb{R}_{+}$. In such a case the following non-uniform counterpart of Lemma 2 can be formulated:

Lemma 3. Assume for each $t_{0} \in \mathbb{R}$ there exist a number $\eta_{t_{0}}>0$ and a continuous function $\varrho_{t_{0}} \in \mathcal{K} \mathcal{L}$ such that for all $p\left(t_{0}\right) \in \mathbb{R}^{l}$ in the system (1) we have (6). Then for every $t_{0} \in \mathbb{R}$ system (1) with $b(t) \equiv 0$ for all $t \in \mathbb{R}$ is GES on $\left[t_{0}, \infty\right)$.

Notice that the lemma implies that for every $t_{0} \in \mathbb{R}$ function $R$ satisfies the PE condition on interval $\left[t_{0}, \infty\right)$, that is, there exist positive real numbers $m_{t_{0}}$ and $\theta_{t_{0}}$ such that

$$
\int_{t}^{t+m_{t_{0}}} R(s) R^{\top}(s) d s \geq \theta_{t_{0}} I_{l}
$$

for all $t \geq t_{0}$. This condition is stronger than (5).

Remark 2. Notice that in Theorem 1 and this lemma, the stability conditions of the system (1) are dependent on $t_{0} \in$ $\mathbb{R}$ (the function $\varrho_{t_{0}}$ and the parameter $\eta_{t_{0}}$ ). Hence, in both cases we have stability properties which are, in general, non uniform with respect to $t_{0} \in \mathbb{R}$, and there is no contradiction with Lemma 2.

\section{B. Robustness with respect to measurement noise}

The conditions of ISS obtained for (1) in Theorem 1 are rather restrictive and related with PE property of the matrix $R$, in the case when $b$ in (1) plays the role of exogenous disturbance. These conditions can be significantly relaxed considering the case with $b$ representing a measurement noise:

$$
\dot{p}(t)=-\gamma R(t) R^{\top}(t)(p(t)+b(t)), t_{0} \in \mathbb{R}, \gamma>0,
$$

where again $p(t) \in \mathbb{R}^{l}$ is the state, the functions $R: \mathbb{R} \rightarrow$ $\mathbb{R}^{l \times k}$ and $b: \mathbb{R} \rightarrow \mathbb{R}^{l}$ are (Lebesgue) measurable and essentially bounded. For all $k \geq 0$ denote a sequence

$$
\phi_{k}=\operatorname{ess}_{t \in\left[t_{k}, t_{k+1}\right)}|R(t)|^{2},
$$

then $\rho_{k} \leq \ell_{k} \phi_{k}$.

Corollary 3. Consider the system (8) satisfying Assumption 1 with an additional constraint:

$$
t_{k+1}=t_{k}+\ell_{k}
$$

for all $k \geq 0$, and assume that the property (5) is valid. If

$$
\begin{gathered}
\gamma \sup _{k \geq 0}\left\{\ell_{k} \phi_{k} e^{\gamma \rho_{k}}+\sum_{i=0}^{k} \prod_{j=i}^{k} \sqrt{1-\frac{\vartheta_{j}}{\gamma^{-1}+\gamma \rho_{j}^{2}}} \ell_{i} \phi_{i} e^{\gamma \rho_{i}}\right\} \\
\leq \eta_{t_{0}}<+\infty
\end{gathered}
$$

then for each $t_{0} \in \mathbb{R}$ there exists a function $\varrho_{t_{0}} \in \mathcal{K} \mathcal{L}$ such that for all $p\left(t_{0}\right) \in \mathbb{R}^{l}$ the estimate (6) is satisfied.

If

$$
\gamma \sup _{k \geq 0} \phi_{k} e^{\gamma \rho_{k}} \leq \beta_{t_{0}}<+\infty
$$


then for each $t_{0} \in \mathbb{R}$ there exists a function $\varrho_{t_{0}} \in \mathcal{K} \mathcal{L}$ such that for all $p\left(t_{0}\right) \in \mathbb{R}^{l}$ the estimate (7) is satisfied.

Recall that the conclusion of Lemma 2 cannot be applied here for the ISS case, since the system (8) in not in the form of (1).

\section{SCALAR CASE}

To illustrate the proposed in Theorem 1 result let us analyze the case with $l=1$.

First, consider the system (1), then solutions can be calculated analytically for all $t \geq t_{0}$ :

$$
p(t)=e^{-\gamma \int_{t_{0}}^{t} R^{2}(\tau) d \tau} p\left(t_{0}\right)+\int_{t_{0}}^{t} e^{-\gamma \int_{s}^{t} R^{2}(\tau) d \tau} b(s) d s
$$

and

$$
|p(t)| \leq e^{-\gamma \int_{t_{0}}^{t} R^{2}(\tau) d \tau}\left|p\left(t_{0}\right)\right|+\int_{t_{0}}^{t}|b(s)| d s .
$$

Under conditions of Theorem 1 we obtain for all $k \geq 0$ :

$$
\left|p\left(t_{k+1}\right)\right| \leq e^{-\gamma \vartheta_{k}}\left|p\left(t_{k}\right)\right|+\int_{t_{k}}^{t_{k+1}}|b(s)| d s,
$$

or for an essentially bounded input $b$ :

$$
\left|p\left(t_{k+1}\right)\right| \leq e^{-\gamma \vartheta_{k}}\left|p\left(t_{k}\right)\right|+\ell_{k}\|b\| .
$$

Recursively applying the above inequalities we get for any $K \geq 0$ :

$$
\left|p\left(t_{K}\right)\right| \leq e^{-\gamma \sum_{k=0}^{K-1} \vartheta_{k}}\left|p\left(t_{0}\right)\right|+\int_{t_{0}}^{t_{K}}|b(s)| d s
$$

or

$$
\left|p\left(t_{K}\right)\right| \leq e^{-\gamma \sum_{k=0}^{K-1} \vartheta_{k}}\left|p\left(t_{0}\right)\right|+\sum_{k=0}^{K-1} \ell_{k} e^{-\gamma \sum_{s=k+1}^{K-1} \vartheta_{s}}\|b\|,
$$

which results in the following condition of the system convergence for the case $b=0$ :

$$
\sum_{k=0}^{+\infty} \vartheta_{k}=+\infty
$$

and the asymptotic gain property is satisfied for any $t_{0} \in \mathbb{R}$ if

$$
\sup _{k \geq 1} \sum_{i=0}^{k-1} \ell_{i} e^{-\gamma \sum_{s=i+1}^{k-1} \vartheta_{s}} \leq \eta\left(t_{0}\right)<+\infty .
$$

These estimates imply that a kind of iISS property is always presented in this case for asymptotically stable systems (1).

Example 1. Select

$$
R(t)=\frac{e^{t} \cos (t)}{1+e^{t}}, b(t)=\sin (t), \gamma=1, p\left(t_{0}\right)=1
$$

and impose

$$
\ell_{k}=\bar{\ell}=0.25 \quad \forall k \geq 0,
$$
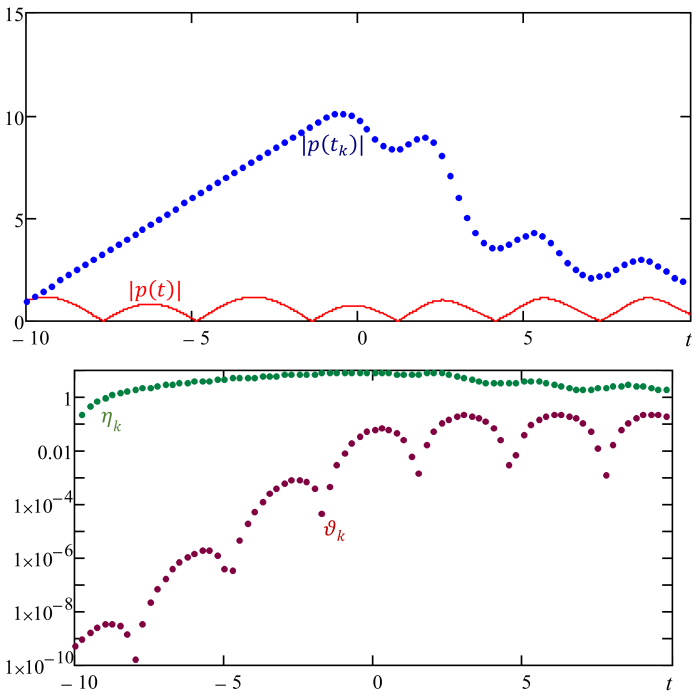

Figure 1. The results of simulation for (1) with $t_{0}=-10$
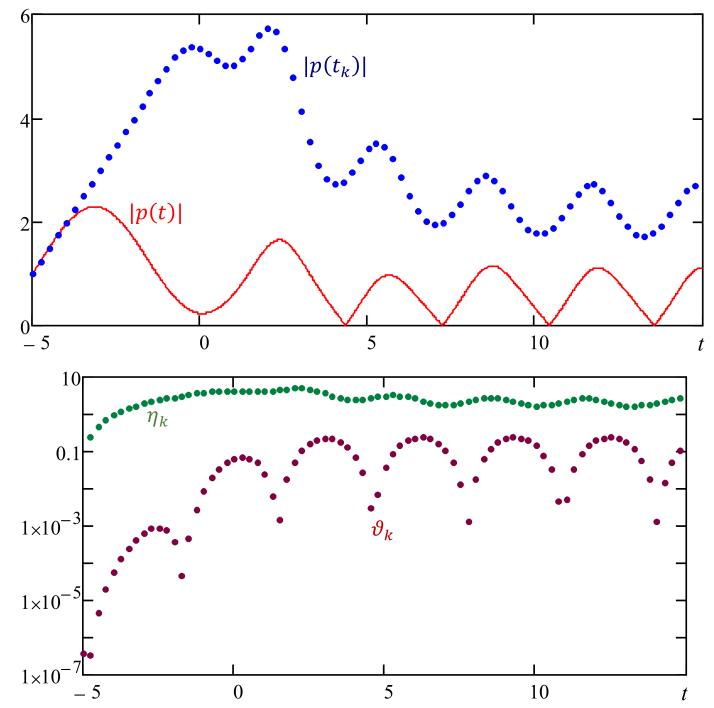

Figure 2. The results of simulation for (1) with $t_{0}=-5$

then the plot of $|p(t)|$ and the estimate on $\left|p\left(t_{k}\right)\right|$ from (9), together with

$$
\vartheta_{k}=\int_{t_{k}}^{t_{k+1}} R^{2}(t) d t, \eta_{k}=\sum_{i=0}^{k-1} \ell_{i} e^{-\gamma \sum_{s=i+1}^{k-1} \vartheta_{s}}
$$

are shown in figures 1 and 2 for $t_{0}=-10$ and $t_{0}=-5$, respectively. These plots demonstrate dependence of $\eta$ on $t_{0}$, and that the system is not uniformly GES (it is GAS) and possesses certain robustness against external inputs, as it has been established in Theorem 1 .

Second, consider the system (8) and for simplicity assume that

$$
\sup _{k \geq 0} \phi_{k} \leq \bar{\phi}<+\infty
$$



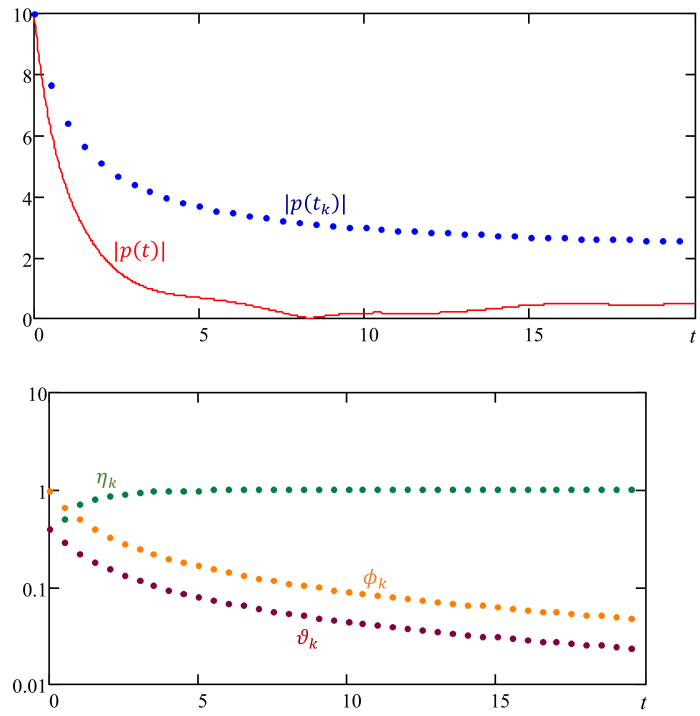

Figure 3. The results of simulation for (8) with $t_{0}=0$

then for any $K \geq 0$ :

$$
\left|p\left(t_{K}\right)\right| \leq e^{-\gamma \sum_{k=0}^{K-1} \vartheta_{k}}\left|p\left(t_{0}\right)\right|+\gamma \bar{\phi} \int_{t_{0}}^{t_{K}}|b(s)| d s
$$

or

$$
\left|p\left(t_{K}\right)\right| \leq e^{-\gamma \sum_{k=0}^{K-1} \vartheta_{k}}\left|p\left(t_{0}\right)\right|+\gamma \sum_{k=0}^{K-1} \phi_{k} \ell_{k} e^{-\gamma \sum_{s=k+1}^{K-1} \vartheta_{s}}\|b\|,
$$

and the ISS property can be observed under the same restrictions as for the case of (1):

$$
\sum_{k=0}^{+\infty} \vartheta_{k}=+\infty, \sup _{k \geq 1} \sum_{i=0}^{k-1} \phi_{i} \ell_{i} e^{-\gamma \sum_{s=i+1}^{k-1} \vartheta_{s}}<+\infty,
$$

while iISS property is naturally yielded for any asymptotically stable system (8).

Example 2. Select $t_{0} \geq 0$,

$$
R(t)=\frac{1}{\sqrt{1+t}}, b(t)=1+\sin (t), \gamma=1, p\left(t_{0}\right)=10,
$$

then the system is not exponentially stable (i.e. $p(t)=$ $p\left(t_{0}\right) \frac{1+t_{0}}{1+t}$ for $t \geq t_{0} \geq 0$ with $\left.b(t)=0\right)$, and impose

$$
\ell_{k}=\bar{\ell}=0.5 \quad \forall k \geq 0 .
$$

The plot of $|p(t)|$ and the estimate on $\left|p\left(t_{k}\right)\right|$ from (10), together with

$$
\begin{gathered}
\vartheta_{k}=\int_{t_{k}}^{t_{k+1}} R^{2}(t) d t, \phi_{k}=\sup _{t \in\left[t_{k}, t_{k+1}\right)}|R(t)|^{2}, \\
\eta_{k}=\sum_{i=0}^{k-1} \phi_{i} \ell_{i} e^{-\gamma \sum_{s=i+1}^{k-1} \vartheta_{s}}
\end{gathered}
$$

are shown in Fig. 3 for $t_{0}=0$.

\section{CONCLUSION}

For a time-varying linear system under a relaxed notion of PE, several relations with ISS and iISS properties are established. It is shown that iISS is rather natural and it is satisfied almost always in this class of systems, and especially with respect to measurement noises. All proposed conditions are sufficient, and investigation of necessary counterpart can be a direction of future research.

\section{REFERENCES}

[1] S. Sastry and M. Bodson, Adaptive Control: Stability, Convergence and Robustness. London: Prentice-Hall, 1989.

[2] H. K. Khalil, Nonlinear Systems, 3rd ed. Upper Saddle River, New Jersey: Prentice Hall, 2002.

[3] L. Praly, "Convergence of the gradient algorithm for linear regression models in the continuous and discrete time cases," December 262016 , int. Rep. MINES ParisTech, Centre Automatique et Systèmes. [Online]. Available: https://hal.archives-ouvertes.fr/hal-01423048

[4] N. Barabanov, R. Ortega, and J. Wang, "On global asymptotic stability of $\dot{x}=-\phi(t) \phi^{\top}(t) x$ with $\phi(t)$ bounded and not persistently exciting," Systems \& Control Letters, vol. 109, pp. 24-27, 2017.

[5] E. Sontag and Y. Wang, "On characterizations of the input-to-state stability property," Systems \& Control Letters, vol. 24, pp. 351-359, 1995.

[6] D. Angeli, E. Sontag, and Y. Wang, "Further equivalencies and semiglobal versions of integral input to state stability," Dynamics and Control, vol. 10 , no. 2 , pp. 127-149, 2000 .

[7] D. Efimov and A. Fradkov, "Design of impulsive adaptive observers for improvement of persistency of excitation," Int. J. Adaptive Control and Signal Processing, vol. 29, no. 6, pp. 765-782, 2015.

[8] I. Karafyllis and J. Tsinias, "Nonuniform in time input-to-state stability and the small gain theorem," IEEE Transactions on Automatic Control, vol. 49, pp. 196-216, 2004.

[9] A. Morgan and K. Narendra, "On the uniform asymptotic stability of certain linear nonautonomous differential equations," SIAM J. Control and Optimization, vol. 15, no. 1, pp. 5-24, 1977. 